\title{
A FUNDAMENTAL THEOREM OF HOMOMORPHISMS FOR SEMIRINGS
}

PAUl J. ALLEN

1. Introduction. When studying ideal theory in semirings, it is natural to consider the quotient structure of a semiring modulo an ideal. If $I$ is an ideal in a semiring $R$, the collection $\{x+I\}_{x \in R}$ of sets $x+I=\{x+i \mid i \in I\}$ need not be a partition of $R$. Faced with this problem, S. Bourne [1], D. R. La Torre [3] and M. Henriksen [2] used equivalence relations to determine cosets relative to an ideal. La Torre successfully established analogues of several well-known isomorphism theorems for rings. However, the methods that Bourne and La Torre used to construct quotient structures proved to be unsuccessful when trying to obtain an exact analogue of the Fundamental Theorem of Homomorphisms.

In this paper, the notion of a $Q$-ideal will be defined and a construction process will be presented by which one can build the quotient structure of a semiring modulo a $Q$-ideal. Maximal homomorphisms will be defined and examples of such homomorphisms will be given. Using these notions, the Fundamental Theorem of Homomorphisms will be generalized to include a large class of semirings.

2. Fundamentals. There are many different definitions of a semiring appearing in the literature. Throughout this paper, a semiring will be defined as follows:

Definition 1. A set $R$ together with two associative binary operations called addition and multiplication (denoted by + and $\cdot$, respectively) will be called a semiring provided:

(i) addition is a commutative operation,

(ii) there exist $0 \in R$ such that $x+0=x$ and $x 0=0 x=0$ for each $x \in R$, and

(iii) multiplication distributes over addition both from the left and from the right.

Definition 2. A subset $I$ of a semiring $R$ will be called an ideal if $a, b \in I$ and $r \in R$ implies $a+b \in I, r a \in I$ and $a r \in I$.

Definition 3. A mapping $\eta$ from the semiring $R$ into the semiring $R^{\prime}$ will be called a homomorphism if $(a+b) \eta=a \eta+b \eta$ and $(a b) \eta=a \eta b \eta$ for each $a, b \in R$. An isomorphism is a one-to-one homomorphism. The

Presented to the Society, January 25, 1968 under the title Quotient structure of a semiring; received by the editors July 1,1968 . 
semirings $R$ and $R^{\prime}$ will be called isomorphic (denoted by $R \cong R^{\prime}$ ) if there exists an isomorphism from $R$ onto $R^{\prime}$.

3. Quotient structures. The notion of a $Q$-ideal will now be defined and a construction process will be presented by which one can build the quotient structure of a semiring with respect to a $Q$-ideal.

Definition 4. An ideal $I$ in the semiring $R$ will be called a $Q$-ideal if there exists a subset $Q$ of $R$ satisfying the following conditions:

(1) $\{q+I\}_{q \in Q}$ is a partition of $R$; and

(2) if $q_{1}, q_{2} \in Q$ such that $q_{1} \neq q_{2}$, then $\left(q_{1}+I\right) \cap\left(q_{2}+I\right)=\varnothing$.

It is clear that every ring ideal $I$ in a ring $R$ is a $Q$-ideal. The following examples will show that $Q$-ideals do occur in semirings that are not rings.

EXAmple 5. Let $R$ be a nonempty, well ordered set and define $a+b=\max (a, b)$ and $a b=\min (a, b)$ for each $a, b \in R . R$ together with the two defined operations forms a semiring. If $r \in R$, then the set $I_{r}=\{x \in R \mid x \leqq r\}$ is an ideal in $R$. It is clear from the definition of addition in $R$ that $0+I_{r}=I_{r}$ and $x+I_{r}=\{x\}$ for each $x>r$. Thus, $I_{r}$ is a $Q$-ideal when $Q=\{0\} \cup\{x \in R \mid x>r\}$.

EXAмple 6. Let $Z_{+}$denote the semiring of nonnegative integers with the usual operations of addition and multiplication. If $m \in Z_{+}-\{0\}$, the ideal $(m)=\left\{n m \mid n \in Z_{+}\right\}$is a $Q$-ideal when $Q=\{0,1, \cdots, m-1\}$. If $m=0$, the ideal $(m)$ is a $Q$-ideal when $Q=Z_{+}$. A simple argument will show the ideal $Z_{+}-\{1\}$ can not be a $Q$-ideal.

Lemma 7. Let I be a $Q$-ideal in the semiring $R$. If $x \in R$, then there exists a unique $q \in Q$ such that $x+I \subset q+I$.

Proof. Let $x \in R$. Since $\{q+I\}_{q \in Q}$ is a partition of $R$, there exists $q \in Q$ such that $x \in q+I$. If $y \in x+I$, there exists $i_{1} \in I$ such that $y=x+i_{1}$. Since $x \in q+I$, there exists $i_{2} \in I$ such that $x=q+i_{2}$. Clearly, $y=x+i_{1}=\left(q+i_{2}\right)+i_{1}=q+\left(i_{2}+i_{1}\right) \in q+I$. Thus, $x+I \subset q+I$. The uniqueness is an immediate result of part (2) of Definition 4.

Let $I$ be a $Q$-ideal in the semiring $R$. In view of the above result, one can define the binary operations $\oplus_{Q}$ and $\odot_{Q}$ on $\{q+I\}_{Q \in Q}$ as follows:

(1) $\left(q_{1}+I\right) \oplus_{Q}\left(q_{2}+I\right)=q_{3}+I$ where $q_{3}$ is the unique element in $Q$ such that $q_{1}+q_{2}+I C q_{3}+I$; and

(2) $\left(q_{1}+I\right) \odot_{Q}\left(q_{2}+I\right)=q_{3}+I$ where $q_{3}$ is the unique element in $Q$ such that $q_{1} q_{2}+I \subset q_{3}+I$. The elements $q_{1}+I$ and $q_{2}+I$ in $\{q+I\}_{q \in Q}$ will be called equal (denoted by $q_{1}+I=q_{2}+I$ ) if and only if $q_{1}=q_{2}$.

THEOREM 8. If $I$ is a $Q$-ideal in the semiring $R$, then 


$$
\left(\{q+I\}_{Q \in Q}, \oplus_{Q}, \odot_{Q}\right)
$$

is a semiring.

Proof. It is an easy matter to show that $\oplus_{Q}$ and $\odot_{Q}$ are associative operations, $\oplus_{Q}$ is a commutative operation, and $\odot_{Q}$ distributes over $\oplus_{Q}$ both from the left and from the right. Define $\phi: R \rightarrow\{q+I\}_{q \in Q}$ by $x \phi=q+I$ where $q$ is the unique element in $Q$ such that $x+I \subset q+I$. It can be shown that $\phi$ is a homomorphism from the semigroup $(R,+)$ onto the semigroup $\left(\{q+I\}_{q \in Q}, \oplus_{Q}\right)$ and $\phi$ is a homomorphism from the semigroup $(R, \cdot)$ onto the semigroup $\left(\{q+I\}_{q \in Q}, \odot_{Q}\right)$. Since 0 is the identity in $(R,+)$, it follows that $0 \phi=q^{*}+I$ is the identity in $\left(\{q+I\}_{q \in Q}, \oplus_{Q}\right)$. Let $q \in Q$ and let $x \in R$ such that $x \phi=q+I$. Since $x 0=0 x=0$, it is clear that $q^{*}+I=0 \phi$ $=(0 x) \phi=0 \phi x \phi=\left(q^{*}+I\right) \quad \odot_{Q}(q+I)$ and $q^{*}+I=0 \phi=(x 0) \phi=x \phi 0 \phi$ $=(q+I) \odot_{Q}\left(q^{*}+I\right)$. Thus, the element $q^{*}+I$ satisfies condition (ii) in Definition 1.

THEOREM 9. Let $I$ be an ideal in the semiring $R$. If $Q_{1}$ and $Q_{2}$ are subsets of $R$ such that $I$ is both a $Q_{1}$-ideal and a $Q_{2}$-ideal, then

$$
\left(\{q+I\}_{q \in Q_{1}}, \oplus_{Q_{1}}, \odot_{Q_{1}}\right) \cong\left(\{q+I\}_{q \in Q_{2}}, \oplus_{Q_{2}}, \odot_{Q_{2}}\right) .
$$

Proof. Define $\eta:\{q+I\}_{q \in Q_{1}} \rightarrow\{q+I\}_{q \in Q_{2}}$ as follows: If $q_{1} \in Q_{1}$, then $\left(q_{1}+I\right) \eta=q_{2}+I$ where $q_{2}$ is the unique element in $Q_{2}$ such that $q_{1}+I \subset q_{2}+I$. It can be shown that $\eta$ is an isomorphism from the semiring $\left(\{q+I\}_{q \in Q_{1}}, \oplus_{Q_{1}}, \odot_{Q_{1}}\right)$ onto the semiring

$$
\left(\{q+I\}_{q \in Q_{2}}, \oplus_{Q_{2}}, \odot_{Q_{2}}\right) .
$$

If $I$ is an ideal in the semiring $R$, then it is possible that $I$ can be considered to be a $Q$-ideal with respect to many different subsets $Q$ of $R$. However, the preceding theorem implies that the structure $\left(\{q+I\}_{Q \in Q}, \oplus_{Q}, \odot_{Q}\right)$ is "essentially independent" of the choice of $Q$. Thus, if $I$ is a $Q$-ideal in $R$ the semiring $\left(\{q+I\}_{Q \in Q}, \oplus_{Q}, \odot_{Q}\right)$ will be denoted by $R / I$ or $(R / I, \oplus, \odot)$.

\section{Maximal homomorphisms.}

Definition 10. A homomorphism $\eta$ from the semiring $R$ onto the semiring $R^{\prime}$ is said to be maximal if for each $a \in R^{\prime}$ there exists $c_{a} \in \eta^{-1}(\{a\})$ such that $x+\operatorname{ker}(\eta) \subset c_{a}+\operatorname{ker}(\eta)$ for each $x \in \eta^{-1}(\{a\})$, where $\operatorname{ker}(\eta)=\{x \in R \mid x \eta=0\}$.

If $\eta$ is a homomorphism from a ring $R$ onto a ring $R^{\prime}$, it is well known that $x+\operatorname{ker}(\eta)=y+\operatorname{ker}(\eta)$ for each $x, y \in \eta^{-1}(\{a\}), a \in R^{\prime}$. Thus, any ring homomorphism is a maximal homomorphism. Unfor- 
tunately, the following example shows that semiring homomorphisms need not be maximal.

EXAMPLE 11. The set $R$ of nonnegative integers is well ordered under the usual ordering of the integers. Thus, $R$ can be considered to be a semiring as described in Example 5. Clearly, $R^{\prime}=\{0,1\}$ is a subsemiring of $R$. Define $\eta: R \rightarrow R^{\prime}$ by $x \eta=0$ if $x \leqq 5$ and $x \eta=1$ if $x>5$. It can be shown that $\eta$ is a homomorphism from $R$ onto $R^{\prime}$. Since $\operatorname{ker}(\eta)=\{x \in R \mid x \leqq 5\}$, it is clear that $y+\operatorname{ker}(\eta)=\{y\}$, for each $y \in \eta^{-1}(\{1\})$. Thus, there does not exist $c_{1} \in \eta^{-1}(\{1\})$ such that $y+\operatorname{ker}(\eta) \subset c_{1}+\operatorname{ker}(\eta)$, for each $y \in \eta^{-1}(\{1\})$.

The following examples will show there exist maximal homomorphisms other than ring homomorphisms.

EXAMPLE 12. The set $R$ of nonnegative real numbers with the usual ordering forms a semiring as described in Example 5. Let $S^{\prime}=\{n / 2 \in R \mid n=1,2,3, \cdots\}$ and $S=\{x \in R \mid 0 \leqq x \leqq 1 / 4\} \cup S^{\prime}$. It is clear that $S$ and $S^{\prime}$ are subsemirings of $R$. If $\eta: S \rightarrow S^{\prime}$ is defined by

$$
\begin{aligned}
& x \eta=0, \quad \text { if } x \in S \text { and } 0 \leqq x \leqq 1 / 4 \text {, } \\
& =n / 2, \quad \text { if } x \in S \quad \text { and } \quad x=n / 2 \text {, }
\end{aligned}
$$

then it can be shown that $\eta$ is a maximal homomorphism.

EXAMPLE 13. Let $Z_{+}$denote the semiring of nonnegative integers described in Example 6, and let $Z /(m)$ denote the ring of integers modulo $(m)$ where $m>0$. If $x \in Z_{+}$, the division algorithm implies there exist unique integers $q$ and $r$ such that $x=q m+r$ where $0 \leqq r<m$. Define $\eta: Z_{+} \rightarrow Z /(m)$ by $x \eta=r+(m)$ where $r$ is the unique integer described above. $\eta$ is a maximal homomorphism from $Z_{+}$onto $Z /(m)$.

5. A fundamental theorem of homomorphisms. Whenever $\eta$ is a maximal homomorphism, $c_{a}$ will denote an element in $\eta^{-1}(\{a\})$ such that $x \in \eta^{-1}(\{a\})$ implies $x+\operatorname{ker}(\eta) \subset c_{a}+\operatorname{ker}(\eta)$. With the aid of this notation and the following lemmas, an analogue of the Fundamental Theorem of Homomorphisms can be obtained.

LEMMA 14. Let $\eta$ be a homomorphism from the semiring $R$ onto the semiring $R^{\prime}$. If $\eta$ is maximal, then $\operatorname{ker}(\eta)$ is a $Q$-ideal, where $Q=\left\{c_{a}\right\}_{a \in R^{\prime}}$.

Proof. It is clear that $U_{a \in R^{\prime}}\left(c_{a}+\operatorname{ker}(\eta)\right)=R$. Let $c_{a}$ and $c_{b}$ be distinct elements in $Q$; i.e., $a \neq b$. Assume $\left(c_{a}+\operatorname{ker}(\eta)\right) \cap\left(c_{b}+\operatorname{ker}(\eta)\right)$ $\neq \varnothing$. Thus, there exist $k, k^{\prime} \in \operatorname{ker}(\eta)$ such that $c_{a}+k=c_{b}+k^{\prime}$. Thus, $a=c_{a} \eta+k \eta=\left(c_{a}+k\right) \eta=\left(c_{b}+k^{\prime}\right) \eta=c_{b} \eta+k^{\prime} \eta=b$, a contradiction. It now follows that $\operatorname{ker}(\eta)$ is a $Q$-ideal. 
Lemma 15. Let $R, R^{\prime}, \eta$ and $Q$ be as stated in Lemma 14, and let $c_{a}$, $c_{b}$ and $c_{c}$ be elements in $Q$.

(1) If $c_{a}+c_{b}+\operatorname{ker}(\eta) \subset c_{c}+\operatorname{ker}(\eta)$, then $a+b=c$.

(2) If $c_{a} c_{b}+\operatorname{ker}(\eta) \subset c_{c}+\operatorname{ker}(\eta)$, then $a b=c$.

Proof. Since $c_{a}+c_{b} \in c_{a}+c_{b}+\operatorname{ker}(\eta) \subset c_{c}+\operatorname{ker}(\eta)$, there exists $k \in \operatorname{ker}(\eta)$ such that $c_{a}+c_{b}=c_{c}+k$. Thus, $a+b=c_{a} \eta+c_{b} \eta=\left(c_{a}+c_{b}\right) \eta$ $=\left(c_{c}+k\right) \eta=c_{c} \eta+k \eta=c$. A similar argument shows (2) is true.

THEOREM 16. If $\eta$ is a maximal homomorphism from the semiring $R$ onto the semiring $R^{\prime}$, then $R / \operatorname{ker}(\eta) \cong R^{\prime}$.

Proof. Define $\bar{\eta}: R / \operatorname{ker}(\eta) \rightarrow R^{\prime}$ by $\left(c_{a}+\operatorname{ker}(\eta)\right) \bar{\eta}=a$, for each $c_{a} \in Q$. It is clear that $\bar{\eta}$ is a one-to-one function from $R / \operatorname{ker}(\eta)$ onto $R^{\prime}$. It will be shown that $\bar{\eta}$ is an isomorphism and the theorem will follow. From the definition of addition in $R / \operatorname{ker}(\eta)$, it follows that $\left[\left(c_{a}+\operatorname{ker}(\eta)\right) \oplus\left(c_{b}+\operatorname{ker}(\eta)\right)\right] \bar{\eta}=\left[c_{c}+\operatorname{ker}(\eta)\right] \bar{\eta}=c$, where $c_{c}$ is the unique element in $Q$ such that $c_{a}+c_{b}+\operatorname{ker}(\eta) \subset c_{c}+\operatorname{ker}(\eta)$. In view of Lemma 15 , it is clear that

$$
\begin{aligned}
& \left(c_{a}+\operatorname{ker}(\eta)\right) \bar{\eta}+\left(c_{b}+\operatorname{ker}(\eta)\right) \bar{\eta} \\
& \quad=a+b=c=\left[\left(c_{a}+\operatorname{ker}(\eta)\right) \oplus\left(c_{b}+\operatorname{ker}(\eta)\right)\right] \bar{\eta} .
\end{aligned}
$$

The definition of multiplication in $R / \operatorname{ker}(\eta)$ implies

$$
\left[\left(c_{a}+\operatorname{ker}(\eta)\right) \odot\left(c_{b}+\operatorname{ker}(\eta)\right)\right] \bar{\eta}=\left[c_{c}+\operatorname{ker}(\eta)\right] \bar{\eta}=c,
$$

where $c_{c}$ is the unique element in $Q$ such that $c_{a} c_{b}+\operatorname{ker}(\eta) \subset c_{c}+\operatorname{ker}(\eta)$. In view of Lemma 15 , it is clear that $\left(c_{a}+\operatorname{ker}(\eta)\right) \bar{\eta}\left(c_{b}+\operatorname{ker}(\eta)\right) \bar{\eta}=a b$ $=c=\left[\left(c_{a}+\operatorname{ker}(\eta)\right) \odot\left(c_{b}+\operatorname{ker}(\eta)\right)\right] \bar{\eta}$.

\section{REFERENCES}

1. S. Bourne, On the homomorphism theorem for semirings, Proc. Nat. Acad. Sci. U.S.A. 38 (1952), 118-119. 321.

2. M. Henriksen, h-ideals in semirings, Notices Amer. Math. Soc. 5 (1958),

3. D. R. La Torre, On h-ideals and k-ideals in hemirings, Publ. Math. Debrecen 12 (1965), 219-226.

University of Alabana 